\title{
Theoretical approach to dependence of crack growth mechanism on confining pressure
}

\author{
H. Kawakata* and M. Shimada \\ Research Center for Earthquake Prediction, Disaster Prevention Research Institute, Kyoto University, Kyoto 611-0011, Japan
}

(Received May 31, 1999; Revised February 7, 2000; Accepted March 7, 2000)

\begin{abstract}
We calculated the stress field on and around an elliptical open crack located in an elastic medium under various confining pressures. The problems were treated as two-dimensional ones by using complex potentials, and the considerable differences between uniaxial and triaxial crack growth mechanisms were recognized. A uniaxial condition allows a certain elliptical open crack to develop by itself (without any crack-crack interactions) along its major semi-axis, whereas a triaxial condition does not. This is the principal mechanism of transition from uniaxial to triaxial crack growth. Our transition model, derived using a theoretical approach, explains the dependence of experimental results, such as crack distribution and internal friction angle, on confining pressure well. We conclude that uniaxial and triaxial crack growth mechanisms fundamentally differ from each other, and that attention must be paid to experimental conditions when applying experimental results to phenomena in the Earth.
\end{abstract}

\section{Introduction}

The mechanism of crack propagation in rocks is an important element in rock mechanics and seismology. A great deal of work has been done since Griffith (1921) proposed the idea of the Griffith crack, and many mathematical problems have been solved, including those related to a single open elliptical crack or cracks of zero thickness (i.e., closed cracks). For example, Jaeger and Cook (1976) gave expressions for the two-dimensional stress and displacement field on and around an elliptical crack. McClintock and Walsh (1962) calculated the conditions for extension of a closed crack, on the surface of which the friction must be taken into account, under biaxial stress, which is known as the modified Griffith theory, and Murrell and Digby (1970a, b) applied this theory to general three-dimensional problems. Various models of wing cracks have been developed to describe the crack propagation (e.g., Horii and Nemat-Nasser, 1985; Jeyakumaran and Rudnicki, 1995; Baud et al., 1996). On the other hand, taking into account the crack closure stress derived by Berg (1965) and Digby and Murrell (1976), some wing cracks will close as the axial stress increases. Further, crack interactions have been studied (e.g., Segall and Pollard, 1980; Lin and Logan, 1991; Lockner and Madden, 1991), which is essential under such condition that a single crack cannot develop by itself.

Attempts have been made to explain the fracture process observed in laboratories in terms of these crack theories. Reches and Lockner (1994) showed experimentally that very-closely-spaced cracking events produce main fractures under a confining pressure of $50 \mathrm{MPa}$. They also ex-

*Now at Geological Survey of Japan/Japan Science and Technology Corporation, Higashi 1-1-3, Tsukuba, Ibaraki 305-8567, Japan.

Copy right (C) The Society of Geomagnetism and Earth, Planetary and Space Sciences (SGEPSS); The Seismological Society of Japan; The Volcanological Society of Japan; The Geodetic Society of Japan; The Japanese Society for Planetary Sciences. plained the fault nucleation process by adopting a theoretical approach to the stress induced by crack opening, although for simplicity their calculations were done assuming zero confining pressure. A uniaxial condition is preferable to a triaxial one for obtaining a high quality data set (i.e., dense data with low noise) of physical properties. Yanagidani et al. (1985) detected a localization of dilatancy by means of dense, high-quality AE measurements in uniaxial compression tests, and Scholz (1990) introduced their results as characteristic of triaxial fracture. However, it is well-known that the fracture mechanism changes drastically with confining pressure (e.g., Wawersik and Brace, 1971). In the present study, assuming a crack in any direction and with any aspect ratio, we examine whether a single crack can develop without any crack interactions under various confining pressures, and discuss the theoretical differences between uniaxial and triaxial conditions. The transitional confining pressure going from a uniaxial condition to a triaxial condition is also given. We also discuss whether open cracks are induced by the opening of a crack under triaxial conditions.

\section{Calculation}

\subsection{Formulation}

In the present study, we consider a two-dimensional problem with a single crack, because we intend to obtain an analytic solution that has a complex variable as a first approximation. In the following sections, the $\mathrm{x}$-axis and $\mathrm{y}$-axis are along the minor and major semi-axes of the elliptical crack, respectively, and their origins are at the center of the elliptical crack (Fig. 1). An elliptical coordinate $\operatorname{system}(\xi, \eta)$ is also used, in which an elliptical crack is represented as $\xi=\xi_{0}$, where $\xi_{0}$ is constant. The angle between the maximum compressional axis and the major semi-axis of the elliptical crack is defined as $\beta$ (Fig. 1). The elliptical crack is supposed to be $2 c \cosh \xi_{0}$ in length. These two coordinate systems are 


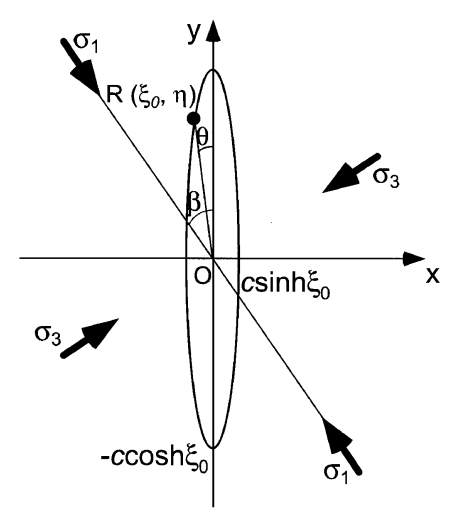

Fig. 1. The coordinate systems used in the present study. In a Cartesian coordinate system, $\mathrm{x}$ - and $\mathrm{y}$-axes are along the minor and the major semi-axes of an assumed elliptical open crack, and the origin is located at the center of the crack. In an elliptical coordinate system, the x-axis is along the surface of the crack, so that the crack surface is represented as $\xi=\xi_{0}$, where $\xi_{0}$ is constant. The origin is also located at the center of the crack. The angles between y-axis and the maximum compressive axis, and between y-axis and the line $O R$, where the point $R$ is the objective point on the crack surface, are $\beta$ and $\theta$, respectively.

related to each other as follows:

$$
\begin{aligned}
& x=c \sinh \xi \sin \eta, \\
& y=c \cosh \xi \cos \eta .
\end{aligned}
$$

The stress components on and around an elliptical crack in the elliptical coordinate system are (Jaeger and Cook, 1976) as follows:

$$
\begin{aligned}
\sigma_{\xi}= & -\Re\left[z^{*} \phi^{\prime \prime}(z)+\psi^{\prime}(z) \frac{\sinh \zeta}{\sinh \zeta^{*}}\right] \\
& +\phi^{\prime}(z)+\phi^{\prime}(z)^{*}, \\
\sigma_{\eta}= & \Re\left[z^{*} \phi^{\prime \prime}(z)+\psi^{\prime}(z) \frac{\sinh \zeta}{\sinh \zeta^{*}}\right] \\
& +\phi^{\prime}(z)+\phi^{\prime}(z)^{*}, \\
\tau_{\xi \eta}= & \Im\left[z^{*} \phi^{\prime \prime}(z)+\psi^{\prime}(z) \frac{\sinh \zeta}{\sinh \zeta^{*}}\right],
\end{aligned}
$$

where $z=y+i x, \zeta=\xi+i \eta(z$ is given by $z=c \cosh \zeta)$ and $\phi$ and $\psi$ are the complex potentials, as follows:

$$
\begin{aligned}
\phi(z)= & \frac{c}{4}\left\{\left(\sigma_{1}-\sigma_{3}\right) e^{2 \xi_{0}} \cos 2 \beta \cosh \zeta\right. \\
& +\left(\sigma_{1}-\sigma_{3}\right) e^{2\left(\xi_{0}+i \beta\right)} \sinh \zeta \\
& \left.+\left(\sigma_{1}+\sigma_{3}\right) \sinh \zeta\right\}, \\
\psi(z)= & -\frac{c}{4} \operatorname{cosech} \zeta\left\{\left(\sigma_{1}+\sigma_{3}\right) \cosh 2 \xi_{0}\right. \\
& +\left(\sigma_{1}-\sigma_{3}\right) e^{2 \xi_{0}} \sinh 2\left(\zeta-\xi_{0}-i \beta\right) \\
& \left.-\left(\sigma_{1}-\sigma_{3}\right) \cos 2 \beta\right\} .
\end{aligned}
$$

In the above expressions, prime, double prime, asterisk, $\Re$, and $\Im$ represent the derivative respect of $z$, the second derivative with respect to $z$, the complex conjugate, the real part, and the imaginary part, respectively. The tangential stress on crack surface $\sigma_{t}$ is given as $\sigma_{t}=\sigma_{\eta}\left(\xi=\xi_{0}\right)$. Jaeger and Cook (1976) also gave the minimum value of $\sigma_{t}$ with respect to $\eta\left(\sigma_{t}\right.$ Min $)$ as follows:

$$
\begin{aligned}
\sigma_{t \mathrm{Min}}= & \frac{1}{\xi_{0}}\left\{\sigma_{1} \sin ^{2} \beta+\sigma_{3} \cos ^{2} \beta\right. \\
& \left.-\left(\sigma_{1}^{2} \sin ^{2} \beta+\sigma_{3}^{2} \cos ^{2} \beta\right)^{\frac{1}{2}}\right\} .
\end{aligned}
$$

In addition, $\sigma_{1}$ and $\sigma_{3}$ represent maximum compressional stress and minimum compressional stress (confining pressure), respectively. As a first approximation, it is assumed that $\sigma_{1}$ and $\sigma_{3}$ satisfy the following empirical relation for the onset of dilatancy for Westerly granite estimated from Brace et al. (1966):

$$
\sigma_{1}=2.64 \sigma_{3}+0.468 C o
$$

in which $C o$ is the uniaxial compressional strength (228 $\mathrm{MPa}$ ).

We applied a criterion for existence of cracks that a crack is squeezed shut if the stress normal to the major semi-axis of the crack exceeds the crack closure stress $\left(\sigma_{\text {close }}\right)$ derived by Berg (1965) as follows:

$$
\sigma_{\text {close }}=G \tanh \xi_{0}(1-v),
$$

where $G$ and $v$ are shear modulus for the elastic medium and Poisson's ratio, respectively. Taking this into account, wing cracks inclined to the maximum compressional axis at about $45^{\circ}$ with a small aspect ratio, which was assumed in previous studies (e.g., Horii and Nemat-Nasser, 1985; Jeyakumaran and Rudnicki, 1995), may close under the triaxial loading condition.

Orowan (1949) and Scholz (1990) gave a criterion for breaking the atomic combination (Orowan's criterion) as follows:

$$
\sigma_{t} \geq E /(2 \pi)
$$

where $E$ is Young's modulus for the medium. If the minimum value of $\sigma_{t}$ Min for a certain crack under some confining pressure satisfies Orowan's criterion, the crack will grow by itself under this condition.

\subsection{Elastic moduli and its dependence on confining pres- sure}

Equations (10) and (11) include the shear modulus, Poisson's ratio, and Young's modulus. The authors and coworkers have carried out laboratory compression test with Westerly granite sample (e.g., Kawakata et al., 1997, 1999), and obtained the values of Shear modulus, Poisson's ratio, and young's modulus measured under confining pressures of $5 \mathrm{MPa}$ and $100 \mathrm{MPa}$ at ambient temperature (Young's modulus was measured also under a confining pressure of 250 $\mathrm{MPa}$ at $150^{\circ} \mathrm{C}$ ), which are listed in Table 1 . Shear modulus and Young's modulus are slightly dependent on confining pressure, while Poisson's ratio shows no confining pressure dependency. Assuming the relationship between the elastic moduli and confining pressure to be linear, the elastic moduli for Westerly granite are given by

$$
\begin{aligned}
G & =18.9 \sigma_{3}+22.2(\mathrm{GPa}), \\
E & =44.0 \sigma_{3}+60.3(\mathrm{GPa}) .
\end{aligned}
$$


Table 1. Young's modulus, Poisson's ratio, and shear modulus of Westerly granite.

\begin{tabular}{cccc}
\hline Confining pressure, MPa & Young's modulus, GPa & Poisson's ratio & Shear modulus, GPa \\
\hline 5 & $60.4 \pm 1.1$ & $0.35 \pm 0.03$ & $22.3 \pm 0.6$ \\
100 & $64.8 \pm 2.9$ & $0.35 \pm 0.03$ & $24.1 \pm 1.0$ \\
$250\left(150^{\circ} \mathrm{C}\right)$ & $71.2 \pm 4.0$ & & \\
\hline
\end{tabular}

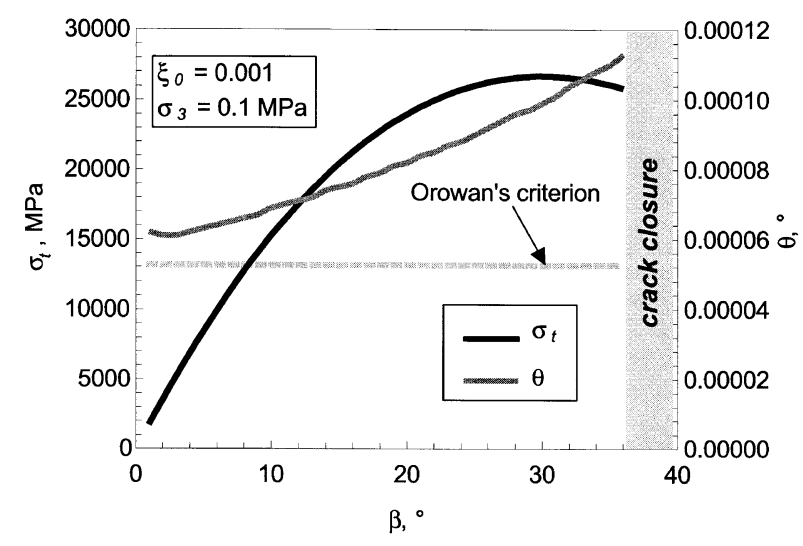

Fig. 2. The tangential stress $\left(\sigma_{t}\right)$ and $\theta$ as a function of $\beta$ under a confining pressure of $0.1 \mathrm{MPa}$. The stress condition is assumed to satisfied the empirical relation for the onset of dilatancy for Westerly granite estimated from Brace et al. (1966), and the aspect ratio of an elliptical open crack $\left(\xi_{0}\right)$ is assumed to be equal to 0.001 .

\section{Calculation Results}

\subsection{Tangential stress field}

Changing $\beta$ and $\xi_{0}$ freely as far as the crack can exist (i.e., the stress normal to the major semi-axis of the crack is smaller than $\sigma_{\text {close }}$ ), the minimum tangential stress on crack surface $\sigma_{t \text { Min }}$ for various confining pressures may be calculated. The minimum value of $\sigma_{t}$ Min is given by Eqs. (8) and (9). Figures 2 and 3 show $\sigma_{t \operatorname{Min}}$ and $\theta$ as a function of $\beta$ (Fig. 1), when $\sigma_{3}$ is equal to $0.1 \mathrm{MPa}$ (atmospheric pressure) and $100 \mathrm{MPa}$, respectively. Under true uniaxial conditions, when $\sigma_{3}=0.1 \mathrm{MPa}$, then $\sigma_{t}$ Min satisfies Orowan's criterion for some $\beta$, for example, $\beta=6^{\circ} \sim 21^{\circ}$ when $\xi_{0}=0.001$ (Fig. 2). On the other hand, $\sigma_{t}$ Min never satisfies Orowan's criterion under a confining pressure of $100 \mathrm{MPa}$. Figure 3 shows the case of $\xi_{0}=0.01$. These results indicate that a certain single elliptical crack grows by itself under uniaxial conditions, but never under triaxial conditions, which may be why the fracture mechanism changes at a particular value of confining pressure. It is sufficient here to discuss the minimum (most tensile) value of tangential stress, because we are concerned with the problem of whether a single crack grows by itself. Figure 4 shows the minimum value of $\sigma_{t \text { Min }}$ and the stress level of Orowan's criterion as a function of confining pressure for Westerly granite. In this case, the transitional confining pressure from the uniaxial fracture to the triaxial one is estimated to be around $20 \mathrm{MPa}$ (Fig. 4). Figure 5 represents $\beta$ and $\theta$ corresponding to $\sigma_{t \text { Min }}$ in Fig. 4 as a function of confining pressure. The expected fracture angle $(=|\beta-\theta|)$ increases as confining pressure is heightened, as is well known.

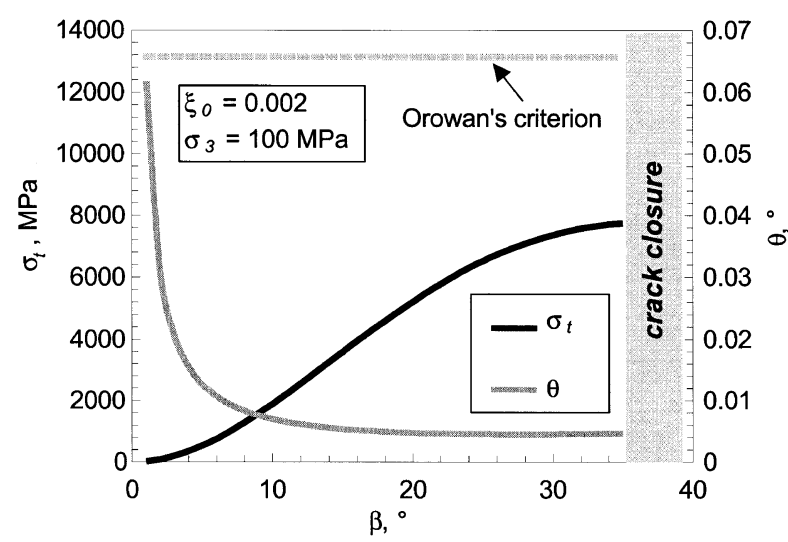

Fig. 3. The same as Fig. 2, except that the value of the confining pressure is $100 \mathrm{MPa}$, and the aspect ratio of an elliptical open crack $\left(\xi_{0}\right)$ is assumed to be equal to 0.002 .

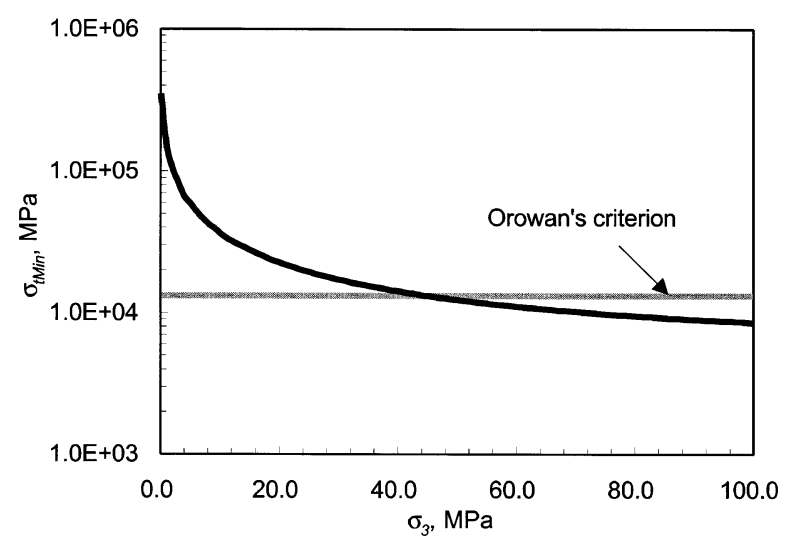

Fig. 4. Relationship between the minimum value of tangential stress ( $\sigma_{t}$ Min $)$ and confining pressure $\left(\sigma_{3}\right)$. The stress condition is assumed to satisfied the empirical relation for the onset of dilatancy for Westerly granite estimated from Brace et al. (1966), and $\xi_{0}$ and $\beta$ are freely changed.

\subsection{Stress components perpendicular to the maximum compressional axis around a crack}

Assuming an elliptical crack parallel to the maximum compressional axis, we calculated the stress components perpendicular to the maximum compressional axis. We assumed that $\sigma_{1}$, and $\sigma_{3}$ were the same as those in the previous section, and that $\xi_{0}$ is equal to 0.01 .

Figure 6(a) represents the stress fields of perpendicular components to the maximum compressional axis under a uniaxial condition. $\beta$ is equal to $0^{\circ}$. The assumed conditions are identical to those of Reches and Lockner (1994), except for the calculation method. As expected, these results 


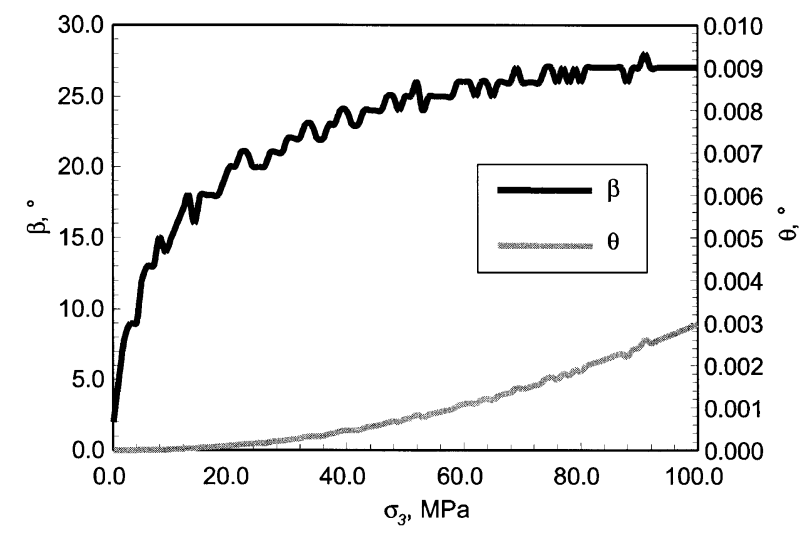

Fig. 5. $\beta$ and $\theta$ as a function of confining pressure $\left(\sigma_{3}\right)$ corresponding to the minimum value of tangential stress $\left(\sigma_{t}\right.$ Min $)$ in Fig. 4.

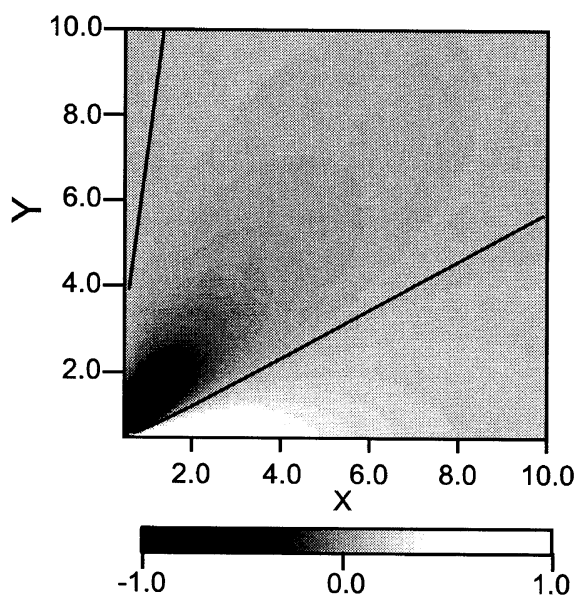

(a)

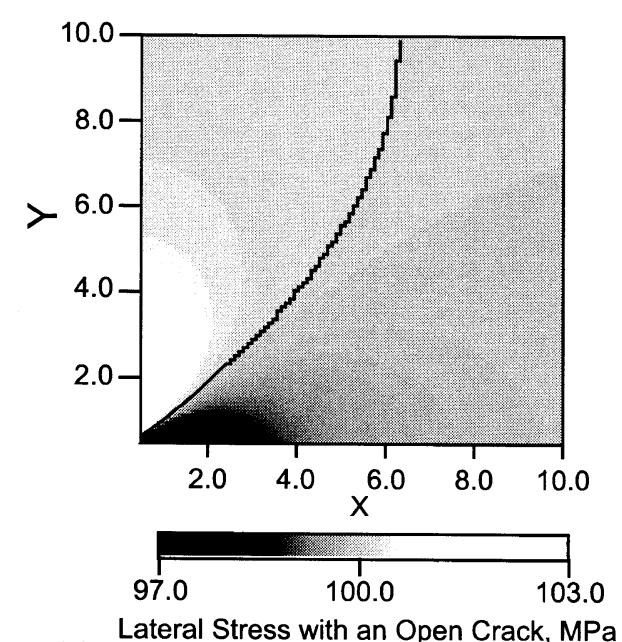

(b)

Fig. 6. Stress field in x-component of around an elliptical open crack under confining pressures of (a) $0.1 \mathrm{MPa}$ and (b) $100 \mathrm{MPa}$. The stress condition is the same as in Figs. 2 and 3, and $\xi_{0}$ and $\beta$ are assumed to be equal to 0.1 and $0^{\circ}$, respectively. Solid curves represent confining pressure.

agree. An open crack along the maximum compressional axis results in the stress field of the perpendicular component ( $\mathrm{x}$-component) being tensile in a near region inclined at about $30^{\circ}$ to the y-axis (Fig. 6(a)). This result supports the idea that an open crack along the maximum compressional axis is likely to open neighboring cracks parallel to it in a direction that is inclined at about $30^{\circ}$ to the maximum compressional axis under uniaxial (atmospheric) conditions, which is consistent with Reches and Lockner (1994).

The features of the stress field change drastically when the confining pressure is $100 \mathrm{MPa}$. Figure 6(b) shows stress fields of the components perpendicular to the maximum compressional axis under a confining pressure of $100 \mathrm{MPa}$ (a triaxial condition). $\beta$ is equal to $0^{\circ}$. In this case, an extensile region of the $\mathrm{x}$-component spreads along the $\mathrm{x}$-axis (Fig. 6(b)), which suggests that an open crack along the maximum compressional axis is likely to open neighboring cracks whose major semi-axes are parallel to its own in a region along the minor semi-axis of the original crack under triaxial conditions. The intensity of heterogeneity is, however, far less than under uniaxial conditions. If we change the confining pressure to $50 \mathrm{MPa}$, the characteristics of the results are almost identical with those under a confining pressure of 100 $\mathrm{MPa}$.

\section{Discussion}

As expressed in the Coulomb criterion, it is known that the fracture angle increases along with the confining pressure. Wawersik and Brace (1971) showed experimentally that the dominant direction of open cracks changes drastically with confining pressure. Kawakata et al. (1999) showed by their experiments with Westerly granite that several fault planes are formed with several steps of stress drop under a confining pressure of $5 \mathrm{MPa}$, while a single fault plane is formed with a single step of stress drop under a confining pressure of 100 $\mathrm{MPa}$ (Figs. 7 and 8). If this transition is fundamental, then analyses and interpretations of experimental results must be done separately for experiments under uniaxial and triaxial conditions. We calculated the tangential stress field on a crack surface, in order to show what this transition mechanism is and whether or not it is fundamental.

Under uniaxial conditions, tangential stress becomes tensile enough to break atomic combinations near the crack tip when a certain open crack exists (Fig. 2). This means that even a single open crack can grow by itself (without any crack-crack interactions) when axial stress reaches a particular value under uniaxial conditions, even if the crack density is not so high. We suggest that this is the principal mechanism of uniaxial fracture. However, it is hard to know which crack grows first in a given rock sample, since many cracks are created under a given compressional condition. In any case, if an open crack starts to grow, it will develop by itself along with a stress drop until it encounters a restraint such as a barrier. In most experiments, platens attached to the top and bottom ends of a sample act as restraints for a developing crack, because of contrasting Young's moduli between the platens and the sample. If the sample is compressed further, then another crack will develop in order to release restored strain energy. These cracks will induce others to open subparallel to them, owing to the extensile stress field that surrounds them (Fig. 5). In such a case, the crack-crack interactions (e.g., Segall and Pollard, 1980; Lin and Logan, 1991; Lockner and Madden, 1991) are important factor to 


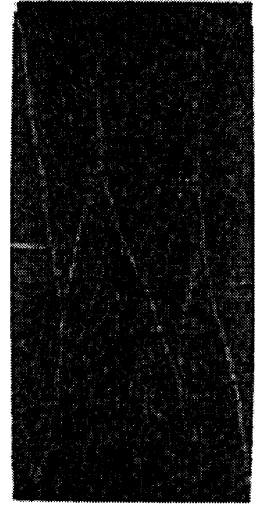

(a)

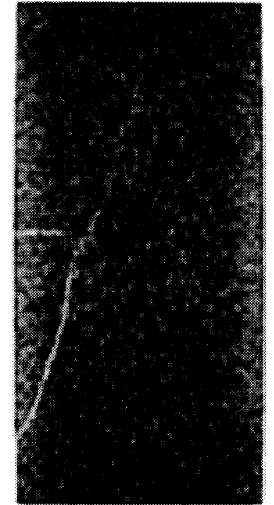

(b)
Fig. 7. Vertical images of sample interior made with an X-ray CT scanning system. The white lines show fault planes inside specimens. Images of Westerly granite samples compressed under a confining pressure of (a) 5 $\mathrm{MPa}$ and (b) $100 \mathrm{MPa}$ (After Kawakata et al., 1999).
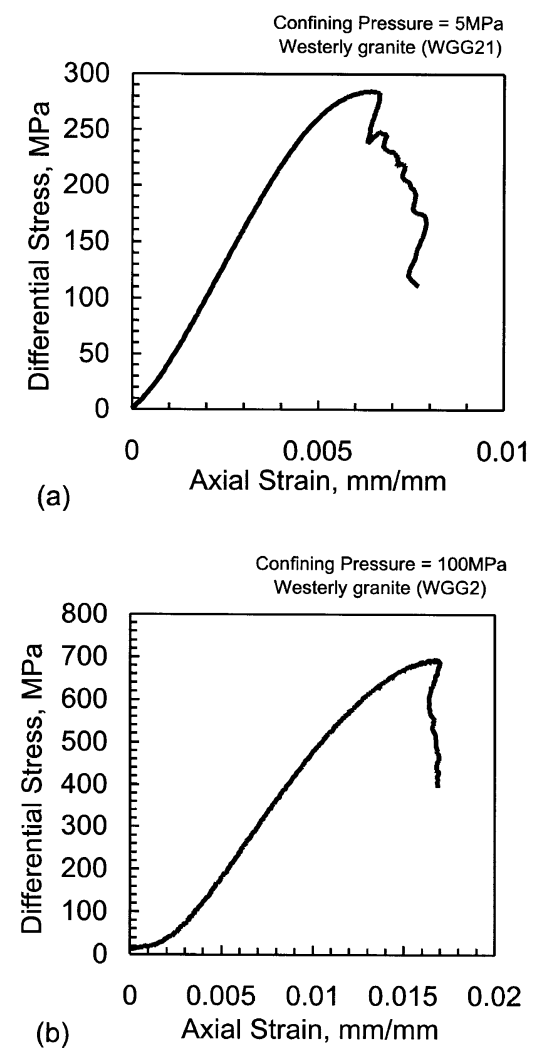

Fig. 8. Relationships between the differential stress and axial strain of Westerly granite compressed under a confining pressure of (a) $5 \mathrm{MPa}$ and (b) $100 \mathrm{MPa}$ (After Kawakata et al., 1999).

describe faulting mechanism. In this way, a complicated system of fault planes will be constructed which meets the maximum compressional axis at a low angle and forms fault zones that include many cracks. These characteristics agree with the experimental results of Wawersik and Brace (1971) and Kawakata et al. (1999).

On the other hand, a single open crack would never develop alone under triaxial conditions, since the tangential stress component is smaller than Orowan's criterion at any point on the crack surface (Fig. 3). Hence, the crack-crack interactions will be the principal mechanism for triaxial fracture, which have been studied in numerous works (e.g., Segall and Pollard, 1980; Lin and Logan, 1991; Lockner and Madden, 1991). Based on the experimental results given by Lockner et al. (1992), a possible mechanism of faulting under triaxial condition is the followings: At an early stage of compression, cracks are distributed uniformly throughout the sample, and crack density becomes higher as axial stress increases. When the crack density in a given part of the sample becomes high enough, then adjacent cracks become connected by intergranular shear cracks. This is the fault nucleation stage accompanied by the localization of crack density. Since shear mode cracking can release more axial strain energy than can tensile mode cracking of the same magnitude, it is difficult for another localization to appear once nucleation occurs. Therefore, a fault plane formed under triaxial conditions is simpler and more inclined to the maximum compressional axis than is one formed under uniaxial conditions.

Previous experimental studies made under triaxial conditions have elucidated many features of the fault formation process. Brace et al. (1966) and Hallbauer et al. (1973) suggested that the coalescence of cracks occurs prior to faulting and that it causes faulting. In addition, Brace et al. (1966), Reches and Lockner (1994), and Kawakata et al. (1997) showed that such prefaulting cracks are localized along a fault plane. Our fault formation mechanism is consistent with all of those, even for the transition from a uniaxial condition to a triaxial one as shown in Wawersik and Brace (1971) and Kawakata et al. (1999). We estimated the transitional confining pressure from the uniaxial fault formation to the triaxial one and obtained a value around $20 \mathrm{MPa}$ for Westerly granite, and Wawersik and Brace (1971) obtained experimentally the value around $35 \mathrm{MPa}$ for Westerly granite, which are consistent.

If the confining pressure is increased further, the fault formation mechanism changes when the compressional strength becomes equal to the frictional strength, which occurs at about $1 \mathrm{GPa}$ of confining pressure for granitic rocks (Shimada and Cho, 1990; Kawakata and Shimada, 1995). This transition cannot be derived by using our calculations, because we assumed the existence of an open crack in the present study. For practical purposes, almost all open cracks will close when the value of the confining pressure exceeds $\sigma_{\text {close }}$. Taking this into account, this transition results from a lack of open cracks, which agrees with experimental results showing that the fault plane is very clear and that shear cracks predominate over tensile cracks (Shimada and Cho, 1990).

\section{Conclusions}

We have calculated the stress fields in an elastic medium, including an elliptical open crack under various values of confining pressure. Under uniaxial conditions, an elliptical open crack subparallel to the maximum compressional axis develops by itself (without any crack-crack interactions) along its major semi-axis because the tangential stress on its surface exceeds the stress level for breaking atomic combinations. It is also likely to open neighboring cracks parallel to it, in a direction inclined at about $30^{\circ}$ to the maximum 
compressional axis. In consequence, fault planes (including adjacent cracks) grow in a direction inclined at a low angle. Under triaxial conditions, on the other hand, any single open crack cannot grow in any direction by itself (without any crack-crack interactions), owing to relatively high tangential stress, so that it cannot result in fault nucleation. As a result, fault nucleation does not occur when the stress field at an open crack is extensile enough to grow by itself, but does occur when crack density in a certain area becomes high enough for cracks to coalesce. Then, the transition from uniaxial to triaxial condition will depend on whether an open crack can grow by itself (without any crack-crack interactions). We estimated the transitional confining pressure from the uniaxial to triaxial condition and obtained a value around $20 \mathrm{MPa}$ for Westerly granite. The single crack growth presented here agrees not only with many aspects reported in previous experimental studies, but provides a possible transition mechanism from uniaxial to triaxial fault formation mechanism.

Finally, we must emphasize that the mechanisms of fault formation under uniaxial and triaxial conditions are fundamentally different.

Acknowledgments. This study was supported in part by a grantin-aid for scientific research (09440159) from the Ministry of Education, Science, Sports and Culture of Japan. We thank Masayuki Kikuchi and an anonymous reviewer for their critical comments and suggestions.

\section{References}

Baud, P., T. Reuschlé, and P. Charlez, An improved wing crack model for the deformation and failure of rock in compression, Int. J. Rock Mech. Min. Sci. and Geomech. Abstr., 33, 539-542, 1996.

Berg, C. A., Deformation of fine cracks under high pressure and shear, $J$. Geophys. Res., 70, 3447-3452, 1965.

Brace, W. F., B. W. Paulding, Jr., and C. H. Scholz, Dilatancy in the fracture of crystalline rocks, J. Geophys. Res., 71, 3939-3953, 1966.

Digby, P. J. and S. A. F. Murrell, The deformation of flat ellipsoidal cavities under large confining pressures, Bull. Seism. Soc. Am., 66, 425-431, 1976.

Griffith, A. A., The phenomena of rupture and flow in solids, Phil. Trans. Roy. Soc. London, Ser. A, Math. Phys. Sci., 211, 163-198, 1921.

Hallbauer, D. K., H. Wagner, and N. G. W. Cook, Some observations concerning the microscopic and mechanical behaviour of quartzite specimens in stiff, triaxial compression tests, Int. J. Rock Mech. Min. Sci. and Geomech. Abstr., 10, 713-726, 1973.
Horii, H. and S. Nemat-Nasser, Compression-induced microcrack growth in brittle solids: Axial splitting and shear failure, J. Geophys. Res., 90, 3105-3125, 1985.

Jaeger, J. C. and N. G. W. Cook, Fundamentals of Rock Mechanics, 2nd ed., 593 pp., Chapman and Hall, London, 1976.

Jeyakumaran, M. and J. W. Rudnicki, The sliding wing crack-Again!, Geophys. Res. Lett., 22, 2901-2904, 1995.

Kawakata, H. and M. Shimada, Frequency-magnitude relation of AE in fracture process of rocks at high confining pressures, Proc. 8th Int. Congr. Rock Mech., 1, 207-210, 1995.

Kawakata, H., A. Cho, T. Yanagidani, and M. Shimada, The observations of faulting in Westerly granite under triaxial compression by X-ray CT scan, Int. J. Rock Mech. Min. Sci., 34: 3/4, Paper No. 151, 1997.

Kawakata, H., A. Cho, T. Kiyama, T. Yanagidani, and M. Shimada, The observations of fault formation in Westerly granite by X-ray CT scan, Tectonophys., 313, 293-305, 1999.

Lin, P. and J. M. Logan, The interaction of two closely spaced cracks: a rock model study, J. Geophys. Res., 96, 21,667-21,675, 1991.

Lockner, D. A. and T. R. Madden, A multiple-crack model of brittle fracture, 1. Non-time-dependent simulations, J. Geophys. Res., 96, 19,62319,642, 1991.

Lockner, D. A., J. D. Byerlee, V. Kuksenko, A. Ponomarev, and A. Sidorin, Observations of quasi-static fault growth from acoustic emissions, in Fault Mechanics and Transport Properties of Rocks, edited by B. Evans and T.-F. Wong, pp. 3-31, Academic Press, San Diego, Calif., 1992.

McClintock, F. A. and J. B. Walsh, Friction on Griffith cracks in rocks under pressure, Proc. 4th U. S. Nat. Congr. Appl. Mech., 2, 1015-1022, 1962.

Murrell, S. A. F. and P. J. Digby, The theory of brittle fracture initiation under triaxial stress conditions - I, Geophys. J. Roy. Astron. Soc., 19, 309-334, 1970a.

Murrell, S. A. F. and P. J. Digby, The theory of brittle fracture initiation under triaxial stress conditions - II, Geophys. J. Roy. Astron. Soc., 19, 499-512, 1970b.

Orowan, E., Fracture and strength of solids, Repts. Prog. Phys., 12, 185-232, 1949.

Reches, Z. and D. A. Lockner, Nucleation and growth of faults in brittle rocks, J. Geophys. Res., 99, 18,159-18,173, 1994.

Scholz, C. H., The Mechanics of Earthquakes and Faulting, 439 pp., Cambridge University Press, Cambridge, 1990.

Segall, P. and D. D. Pollard, Mechanics of discontinuous faults, J. Geophys. Res., 85, 4337-4350, 1980.

Shimada, M. and A. Cho, Two types of brittle fracture of silicate rocks under confining pressure and their implication in the earth's crust, Tectonophys., 175, 221-235, 1990.

Wawersik, W. R. and W. F. Brace, Post-failure behavior of a granite and diabase, Rock Mech., 3, 61-85, 1971.

Yanagidani, T., S. Ehara, O. Nishizawa, K. Kusunose, and M. Terada, Localization of dilatancy in Ohshima granite under constant uniaxial stress, J. Geophys. Res., 90, 6840-6858, 1985.

H. Kawakata (e-mail: kawakata@gsj.go.jp) and M. Shimada 